\title{
Characterization of Medipix3 With Synchrotron Radiation
}

\author{
Eva N. Gimenez, Rafael Ballabriga, Member, IEEE, Michael Campbell, Ian Horswell, Xavier Llopart, \\ Julien Marchal, Kawal J. S. Sawhney, Nicola Tartoni, Member, IEEE, and Daniel Turecek
}

\begin{abstract}
Medipix 3 is the latest generation of photon counting readout chips of the Medipix family. With the same dimensions as Medipix2 $(256 \times 256$ pixels of $55 \mu \mathrm{m} \times 55 \mu \mathrm{m}$ pitch each $)$, Medipix 3 is however implemented in an 8-layer metallization 0.13 $\mu \mathrm{m}$ CMOS technology which leads to an increase in the functionality associated with each pixel over Medipix2. One of the new operational modes implemented in the front-end architecture is the Charge Summing Mode (CSM). This mode consists of a charge reconstruction and hit allocation algorithm which eliminates event-by-event the low energy counts produced by charge-shared events between adjacent pixels. The present work focuses on the study of the CSM mode and compares it to the Single Pixel Mode (SPM) which is the conventional readout method for these kind of detectors and it is also implemented in Medipix3. Tests of a Medipix 3 chip bump-bonded to a $300 \mu \mathrm{m}$ thick silicon photodiode sensor were performed at the Diamond Light Source synchrotron to evaluate the performance of the new Medipix chip. Studies showed that when Medipix 3 is operated in CSM mode, it generates a single count per detected event and consequently the charge sharing effect between adjacent pixels is eliminated. However in CSM mode, it was also observed that an incorrect allocation of $\mathrm{X}$-rays counts in the pixels occurred due to an unexpectedly high pixel-to-pixel threshold variation. The present experiment helped to better understand the CSM operating mode and to redesign the Medipix 3 to overcome this pixel-to-pixel mismatch.
\end{abstract}

Index Terms-Medipix3, synchrotron radiation, X-ray detectors, X-ray imaging.

\section{INTRODUCTION}

$\mathbf{T}$ HE brilliance now being achieved at the 3rd generation of synchrotrons has increased the potential for high quality $\mathrm{X}$-ray diffraction experiments. Hence, new high-performance detectors with a high spatial resolution, very high dynamic range and high detection efficiency are required. Hybrid silicon photon-counting detectors can meet these requirements [1].

Manuscript received June 30, 2010; revised October 07, 2010; accepted October 12, 2010. Date of publication December 03, 2010; date of current version February 09, 2011. This work was supported by Diamond Light Source.

E. N. Gimenez, I. Horswell, J. Marchal, K. J. S. Sawhney, and N. Tartoni are with Diamond Light Source, Harwell Science and Innovation Campus, Didcot, Oxfordshire OX11 0DE, U.K. (e-mail: Eva.Gimenez@diamond.ac.uk; Ian.Horswell@diamond.ac.uk; Julien.Marchal@diamond.ac.uk; Kawal.Sawhney@diamond.ac.uk; Nicola.Tartoni@diamond.ac.uk).

R. Ballabriga, M. Campbell, and X. Llopart are with CERN, European Organization for Nuclear Research, CH-1211 Genève 23, Switzerland (e-mail: Rafael. Ballabriga@cern.ch; Michael.Campbell@cern.ch; Xavier.Llopart@cern.ch).

D. Turecek is with the Institute of Experimental and Applied Physics, Technical University in Prague, 12800 Prague 2, Czech Republic (e-mail: Daniel. Turecek@cern.ch).

Color versions of one or more of the figures in this paper are available online at http://ieeexplore.ieee.org.

Digital Object Identifier 10.1109/TNS.2010.2089062
These detectors normally consist of monolithic arrays of silicon photodiodes bump-bonded to CMOS electronic readout chips. Currently both fields, silicon photodiodes and CMOS technology, are experiencing considerable improvements which are leading to an increase in detector performance. New generations have also emerged in other families of hybrid pixel detectors, such as Pilatus II [2] and XPAD3 [3], [4]. The advantages of these new photon-counting detectors were quickly appreciated in scattering experiments. However, their pixel pitch of $172 \mu \mathrm{m}$ and $130 \mu \mathrm{m}$ respectively, limited their satisfactory use in other experiments as for example with coherent $\mathrm{X}$-ray diffraction. When a survey of the requirements for future detectors was carried out in conjunction with beamline scientists at the Diamond Light Source (DLS) synchrotron [5], the need for detectors with a pixel pitch smaller than $100 \mu \mathrm{m}$ was highlighted with the additional property of being radiation hard [6].

The present work was focused on the new Medipix 3 readout chip [7], [8] which takes advantage of the advances in CMOS technology to allow a high level of functionality to be implemented in each pixel of size $55 \mu \mathrm{m} \times 55 \mu \mathrm{m}$. The Medipix 3 chip enables the development of hybrid pixel detectors which accomplish the aforementioned requirements and can improve the quality of data acquired in synchrotron experiments. The evaluation of the new Medipix3 readout chip was done at beamline B16 [9] of the DLS synchrotron. Experiments were performed in order to study the detector response and its image quality.

\section{MEDIPIX3 DETECTOR}

The detector consisted of a $300 \mu \mathrm{m}$ thick pixel array silicon sensor of resistivity $13 \mathrm{k} \Omega \bullet \mathrm{cm}$ bump-bonded to the Medipix 3 readout chip. The system was operated with a bias voltage of $90 \mathrm{~V}$ resulting in full depletion of the sensor and was readout using Medipix3 USB interfaces and Pixelman software, produced by the IEAP [10], [11].

Medipix 3 readout chip, the latest in the Medipix family, has the same dimensions as Medipix2 $(256 \times 256$ pixels of $55 \mu \mathrm{m} \times 55 \mu \mathrm{m}$ size each) but it is manufactured in an 8-metal $0.13 \mu \mathrm{m}$ CMOS technology which enables further functions to be implemented for each pixel. Amongst others, Medipix 3 has a new front-end architecture aimed at eliminating the spectral distortion produced by the charge sharing seen in highly segmented semiconductor detectors, as observed in the Medipix2 chip [12]-[14]. In the new architecture, the charge generated by a photon is reconstructed by grouping pixels in clusters of four and summing the charge collected in each cluster. The event is then assigned as a single hit to the summing circuit with the largest charge deposit. This operating mode, referred to as Charge Summing Mode (CSM), is complementary to the 
operating mode in conventional detector systems, referred to as Single Pixel Mode (SPM) [15]. The Medipix3 chip may still be programmed for SPM by disabling the charge reconstruction and the communication between neighbouring pixels. Another of the new operational modes enables the size of the pixel readout channel to be configured. It is also possible to group the readout pixels in clusters of four and the cluster to become a single detection unit allowing up to 8 thresholds for energy binning, this is referred to as Spectroscopic Mode (SM). In this mode, the chip should be connected to a sensor with a pixel pitch of $110 \mu \mathrm{m}$.

Each pixel cell of the readout chip occupies an area of $55 \mu \mathrm{m} \times 55 \mu \mathrm{m}$ and contains an analog front-end and digital processing circuitry. The analog front-end consists of a preamplifier, a shaper and two threshold discriminators. The first discriminator is used to define the global lower threshold (THL) and as an input to the arbitration logic when charge summing is enabled. The second discriminator can be used to define a global high threshold (THH). The digital circuitry contains control logic, arbitration modules for hit allocation, circuitry for storage of the pixel configuration data and two registers that can be configured as two 1-bit, 4-bit or 12 bit counters or as a single 24 bit counter. In 24-bit counting mode only one discrimination level is used [16]. Medipix 3 also exhibits two operational gain modes: low gain mode and high gain mode. In the tests performed at the synchrotron, only the high gain mode was used, since the energies of the experiment went from $6 \mathrm{keV}$ to $20 \mathrm{keV}$. Furthermore, techniques were used in the design of the chip in order to minimize the radiation damage.

\section{BEAMLINE SET-UP}

The DLS synchrotron is made up of three stages: a linac, a synchrotron booster and a storage ring. For the tests, the energy of the electrons circulating in the storage ring was $3 \mathrm{GeV}$ and were grouped in bunches $10 \mathrm{ps}$ long with a minimum spacing between contiguous bunches of $2 \mathrm{~ns}$. Up to 936 contiguous bunches can travel in the storage ring of DLS; however generally DLS is operated in gapped mode where not all 936 bunches are present. During our experiment 685 contiguous electron bunches were present in the storage ring and 251 missing. Therefore, the generated X-ray beam had an overall burst duration of $1370 \mathrm{~ns}$ followed by $502 \mathrm{~ns}$ period without photons. The cycle was repeated every $1.872 \mu \mathrm{s}$, which is the revolution period of the electrons in the storage ring of DLS. The storage ring was operated at a current of $150 \mathrm{~mA}$ in top-up mode, i.e. a small amount of electrons were injected every ten minutes to compensate for losses and keep the current constant to within $1 \%$.

Beamline B16 aims at developing novel techniques and characterizing optics, detectors and other instrumentation. It provides both white and monochromatic X-ray beams in the 4-25 keV photon energy range. The Medipix 3 detector test consisted of several experiments. In all of them a monochromatic beam achieved by a $\mathrm{Si}(111)$ double crystal monochromator was used for the characterisation of the detector. Depending on the experiment, the dimension and characteristics of the X-ray beam varied as described in the next subsections.

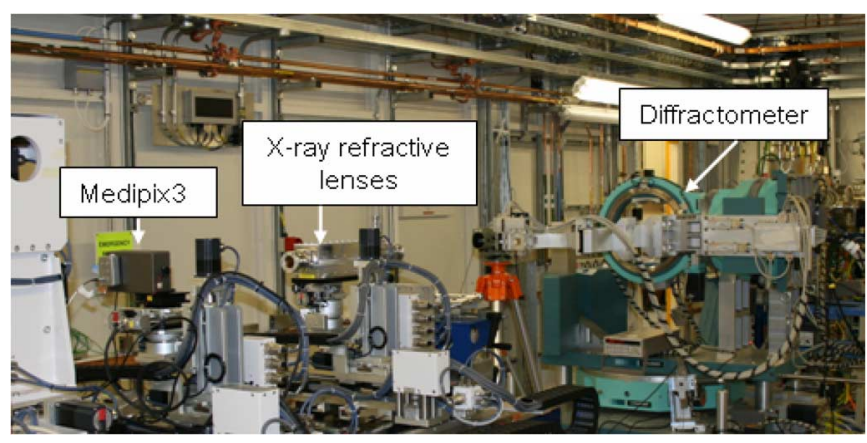

Fig. 1. Experimental set-up at B16 test beamline for monochromatic microfocused beam.

\section{A. Unfocused Monochromatic Beam}

An unfocused monochromatic beam (set at several energies between $6 \mathrm{keV}$ and $20 \mathrm{keV}$ ) was used to study the threshold equalization procedures, the linearity and the homogeneity of the detector's response. Appropriate beam sizes were generated using the beamline slits.

\section{B. Micro-Focused Monochromatic Beam}

A special feature of this beamline is the ability to deliver a micro-focused beam a few micrometers in size. The charge collection and readout process of the CSM and SPM modes of the Medipix 3 detector were studied by scanning the pixels in the detector with a micro-focused X-ray beam. The micro-focused monochromatic beam was generated using a compound refractive lens (CRL) and delivered photons at $15 \mathrm{keV}$. The experimental set-up is shown in Fig. 1. The CRL comprises 98 lenses stacked in line in a He filled chamber. All the lenses were identical: made of beryllium, of parabolic shape, with a radius of $0.2 \mathrm{~mm}$ at the apex and a geometrical aperture of 1 $\mathrm{mm}$. The parabolic shape provides 2 dimensional focusing. The Medipix 3 detector was installed approximately $0.67 \mathrm{~m}$ downstream of the CRL lenses, on a versatile optic table where the detector and CRL lenses were aligned to the synchrotron beam. The size of the micro-focused beam was measured by taking transmission scans of $200 \mu \mathrm{m}$ diameter Au cross-wires. The derivatives of the wire scans gave the beam size. The raw data and the derivative, for vertical and horizontal scans, are shown in Fig. 2. The measured FWHM size of the micro-focused beam was $2.2 \mu \mathrm{m} \times 2.9 \mu \mathrm{m}$ in $x$ and $y$, respectively.

\section{THRESHOLD EOUALIZATION}

The Medipix3 chips contain two 9-bit Digital to Analog Converters (DACs) for setting the global low and high thresholds (THL and THH respectively) used to determine whether or not a hit is registered. However CMOS transistors experience mismatch, which is the process that causes random variations in the physical quantities of identically designed devices. This mismatch causes pixel-to-pixel gain and offset variations in the analog front-end circuits. To correct for this mismatch, threshold equalization needs to be performed before using the detector for measurements. A simplified schematic of the threshold equalization circuitry for SPM mode is shown in Fig. 3. Transistor mismatch in the front-end causes a DC level 


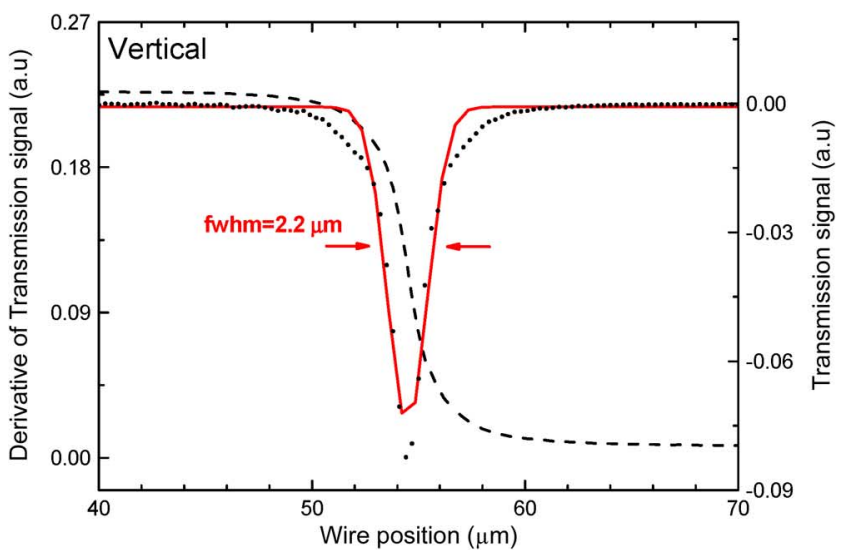

(a)

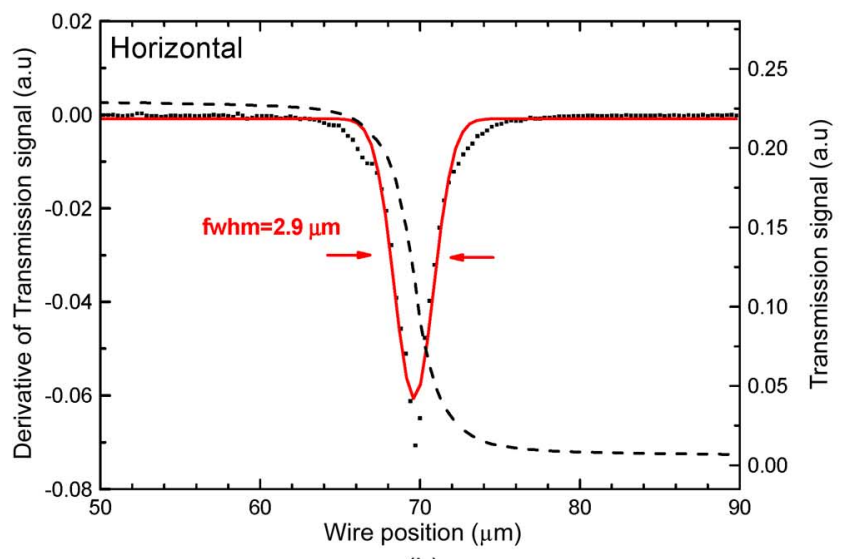

(b)

Fig. 2. Transmission signal from a Au wire scan (dashed line) in the focal plane of the compound refractive micro-focusing lens measured at $15 \mathrm{keV}$ photon energy. The derivative of the raw data (dots) and a Gaussian fit (solid line) are also shown. Plots (a) and (b) are for vertical and horizontal beam profiles, respectively.

shift of the shaper output current whose amplitude contains the information of the induced charge in the pixel input pad. Each pixel of the Medipix3 has DACs to adjust this DC level which connects to the crossing discriminator. The DAC uses 5 equalization bits: one bit controls the $\mathrm{I}_{\mathrm{THN}}$ current source, which is activated when the DC offset is negative; and the other 4 bits are used for threshold trimming by means of the current

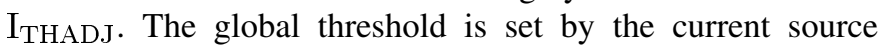
$\mathrm{I}_{\mathrm{THP}}$. Two 8-bit DACs in the periphery are used to generate the bias for the $\mathrm{I}_{\mathrm{THN}}$ and $\mathrm{I}_{\mathrm{THADJ}}$ current sources (THN and DAC_Pixel respectively) [15], [16].

Two threshold equalizations of the pixel matrix for the THL (THH was not used during these tests) were performed: one using the noise edge of the THL threshold scan (see Section IV-A); the other with X-rays of a given energy (see Section IV-B) using the inflexion point of the integral spectrum obtained by plotting the total number of counts when scanning along the THL DAC (referred to as S-curves).

\section{A. Threshold Equalization on Noise}

The THL threshold equalization on noise starts by defining a THL DAC target value where the noise edge of all pixels will be set after threshold tuning. The edge was defined by the first

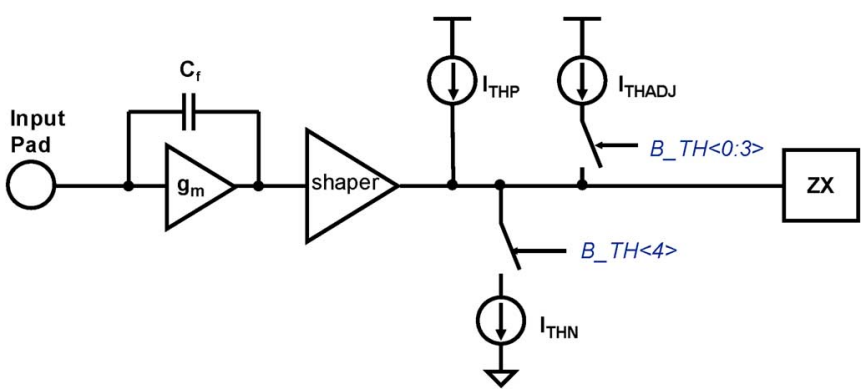

Fig. 3. Schematic of the front-end circuitry in Single Pixel Mode including the threshold adjustment mechanism and the zero-crossing discriminator. $\mathrm{I}_{\mathrm{THP}}$ sets the global threshold. $\mathrm{I}_{\mathrm{THADJ}}$ and $\mathrm{I}_{\mathrm{THN}}$ adjust the threshold in the matrix.

point in the S-curve where each pixel detects three events in the sample time. Subsequent THL threshold scans using the equalization algorithm are used to optimize the settings of two of the periphery DACs (THN DAC and DAC_pixel). After the optimal value of these DACs is found, the on-pixel 5-bit DAC equalization coefficients are tuned to locate the pixel noise edge as close as possible to the THL DAC target value.

\section{B. Threshold Equalization on $8 \mathrm{keV} X$-rays}

In order to flood the detector with X-rays, a $\mathrm{Cu}$ sheet was placed at 45 degrees to the unfocussed monochromatic beam to produce fluorescence X-rays of energy $8 \mathrm{keV}$. The beam energy was tuned to $9.3 \mathrm{keV}$ (above the K-edge of $\mathrm{Cu}$ ). The detector was placed at a distance of $20 \mathrm{~cm}$ from the $\mathrm{Cu}$ target and perpendicular to the direction of the $8 \mathrm{keV} \mathrm{X}$-rays. The threshold equalization followed the same process as the one described is Section IV-A. for the threshold equalization on noise keeping the previously found settings of THN and DAC_pixel periphery DACs. The definition of the initial THL DAC target value was set according to the energy of the incoming radiation, in this case 35 DAC units higher than the noise edge for this energy.

\section{Threshold Equalization Precision in Single Pixel Mode}

The precision of the threshold equalization procedures was investigated by flood illuminating the detectors with $8 \mathrm{keV}$ $\mathrm{X}$-rays from the $\mathrm{Cu}$ sheet and scanning the THL threshold across the X-ray signal down to the noise for the two types of threshold equalization masks loaded in the detector. Fig. 4(a). and Fig. 5(a). show the individual pixel S-curves for the $8 \mathrm{keV}$ $\mathrm{X}$-ray illumination obtained with the equalization on noise and on $8 \mathrm{keV} \mathrm{X}$-rays, respectively. The mean of the selected pixels response is plotted as a black wide line.

In these figures, $\mathrm{S}$-curves of individual pixels are less widely spread around the mean in the region of the inflection point when the detector THL was equalized with X-rays instead of with the noise edge. This is due to the fact that the equalization done with X-rays compensates for gain and offset variations between pixels whereas equalization done on noise compensates only for offset variations.

An estimation of the threshold dispersion over the detector can be obtained by plotting the threshold value corresponding to the edge (see Section VI-A) of the X-ray signal S-curve for each pixel. A histogram of these values is shown in Fig. 4(b). and 


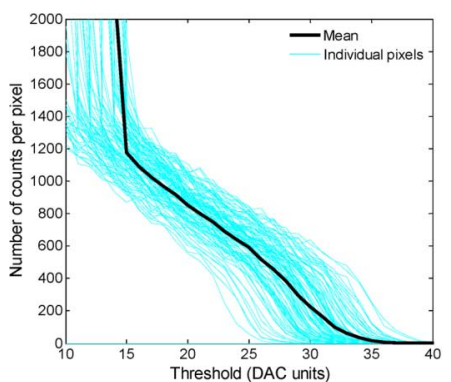

(a)

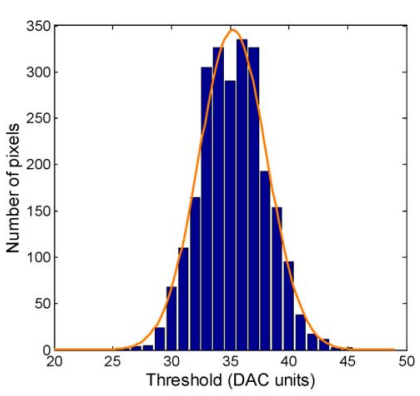

(b)
Fig. 4. THL equalized on the noise edge for a detector operated in Single Pixel Mode. (a) A ROI of $10 \times 10$ individual pixel $S$-curves are displayed around the mean (black line) corresponding to X-ray signal. (b) Distribution of pixel THL values after equalization on noise.

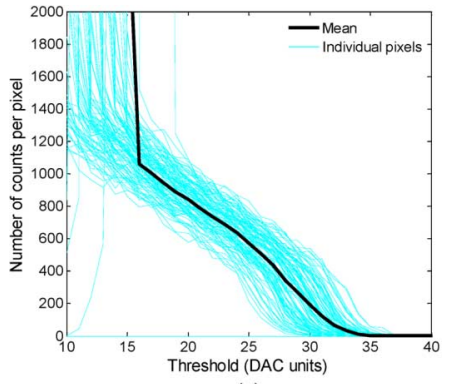

(a)

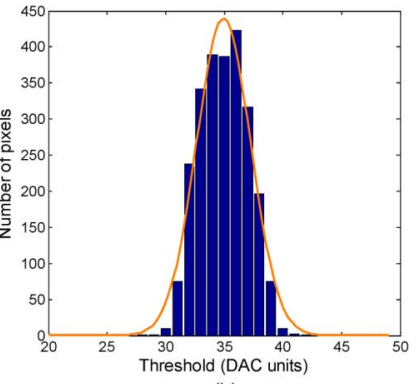

(b)
Fig. 5. THL equalized on $8 \mathrm{keV} \mathrm{X}$-rays edge for a detector operated in Single Pixel Mode. (a) A ROI of $10 \times 10$ individual pixel S-curves are displayed around the mean S-curve (black line) corresponding to X-ray signal. (b) Distribution of pixel THL values after equalization on $8 \mathrm{keV}$ X-ray edge.

TABLE I

STANDARD DEVIATION VALUES $(\sigma)$ OF THE THRESHOLD DisPERSION HISTOGRAM FOR THRESHOLD EQUALIZATION ON NOISE AND ON 8-keV ENERGY PHOTONS IN SPM MODE

\begin{tabular}{cccc}
\hline \hline $\begin{array}{c}\text { Threshold } \\
\text { equalization }\end{array}$ & $\sigma$ (DAC units) & $\sigma(\mathrm{e}-\mathrm{rms})$ & $\sigma(\mathrm{keV})$ \\
\hline On noise & 2.9 & 290 & 1.04 \\
On Cu & 2.3 & 230 & 0.83 \\
\hline \hline
\end{tabular}

Fig. 5(b). A Gaussian distribution was fitted to the threshold dispersion histogram for the threshold equalization on noise and on $8 \mathrm{keV}$ energy photons in SPM. Table I summarizes the standard deviation values of the Gaussian fit. The measured threshold variation was 3.5 times larger than that expected from simulations, this discrepancy is being investigated. The equivalent values in $\mathrm{keV}$ and e-rms were calculated using the conversion factor of $0.36 \mathrm{keV} / \mathrm{DAC}$ step obtained from the energy calibration of the detector which will be discussed in Section V and the energy to electrons conversion factor of $3.6 \mathrm{eV} / \mathrm{e}-\mathrm{hole}$ pair for silicon.

This study was repeated by detecting $16 \mathrm{keV} \mathrm{X}$-rays from a $\mathrm{Zr}$ sheet with each of the equalization masks, on noise and on $8 \mathrm{keV} \mathrm{X}$-rays. The set-up and procedure was the same as the previously described but with a $\mathrm{Zr}$ sheet instead of a $\mathrm{Cu}$ one. Results show a $30 \%$ increase in the dispersion of the pixel THL values for $16 \mathrm{keV} \mathrm{X}$-rays compared to the dispersion measured for $8 \mathrm{keV} \mathrm{X-rays.}$

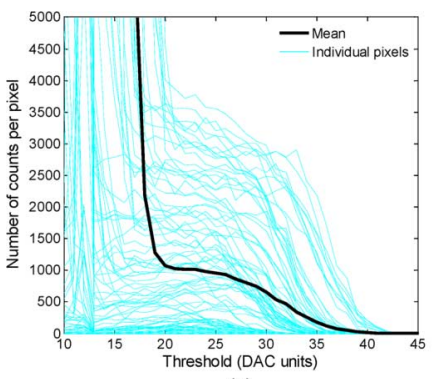

(a)

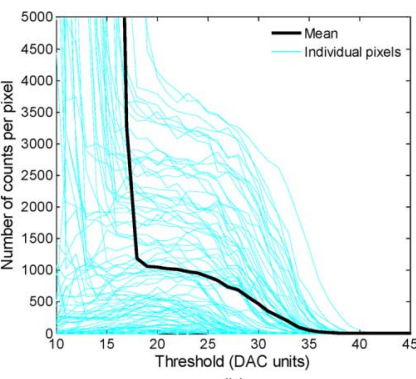

(b)
Fig. 6. A ROI of $10 \times 10$ individual pixel S-curves are displayed around the mean (black line) for THL equalized on the noise edge (a) and $8 \mathrm{keV}$ X-rays edge (b) for a detector operated in Charge Summing Mode.

\section{Threshold Equalization in Charge Summing Mode}

Threshold adjustment was also performed on the noise edge and $\mathrm{Cu} \mathrm{X}$-ray fluorescence edge for the detector operated in CSM mode. Fig. 6. shows the individual pixel S-curves for the $\mathrm{Cu}$ flood illumination obtained after equalization on noise and on $8 \mathrm{keV} \mathrm{X-rays,} \mathrm{respectively.} \mathrm{The} \mathrm{S-curves} \mathrm{for} \mathrm{individual}$ pixels show a larger dispersion in the pixel response compared to SPM mode. This is explained by the observation that some pixels are never collecting any X-rays because their hits are wrongly being allocated to their neighbours (see Section VIII). This effect is present independently of the threshold equalization method used (noise or X-rays). The strong distortion observed in CSM mode further confirms the unexpectedly high component mismatch which was already observed in SPM mode (see Section IV-C).

\section{LINEARITY}

Unfocused monochromatic X-ray beams at $6 \mathrm{keV}, 10 \mathrm{keV}, 15$ $\mathrm{keV}$ and $20 \mathrm{keV}$ photon energies were used to study the linearity of the detector. Additionally, the monochromator was adjusted in order to provide the $3 \mathrm{rd}$ harmonic of these energies. Using these 3rd harmonics, energies at $18 \mathrm{keV}, 30 \mathrm{keV}, 45 \mathrm{keV}$ and 60 $\mathrm{keV}$ were also available. Before acquiring data, the threshold equalization procedure on noise was used to trim the pixel matrix. The same threshold equalization file was used for all energies except for $6 \mathrm{keV}$. For this energy, a new THL threshold equalization on noise was performed, since this energy was very close to the noise edge (see Fig. 7.).

For each one of the aforementioned X-ray energies, a scan of the THL threshold was performed. The total number of counts was calculated for each image (excluding noisy pixels), producing an integral spectrum of counts vs low threshold (THL) setting. Fig. 7. shows the S-curves for $6 \mathrm{keV}, 15 \mathrm{keV}$ and 20 $\mathrm{keV}$ photon energy for SPM and CSM modes. In all energies, there is a slope related to charge-shared events for SPM mode. In CSM mode instead of this slope there is a flat region which implies that the charge sharing effect is being corrected when operating the chip in this mode. Fig. 8. shows the differential spectrum obtained from the S-curves for SPM and CSM modes at 15 $\mathrm{keV}$ energy photons. For SPM mode, additional counts at low energy are observed, which are due to the charge cloud being shared between pixels. Whereas in CSM mode, a clear photo peak spectrum is visible. In this mode, the charge reconstruction and hit allocation algorithm implemented in the front-end 


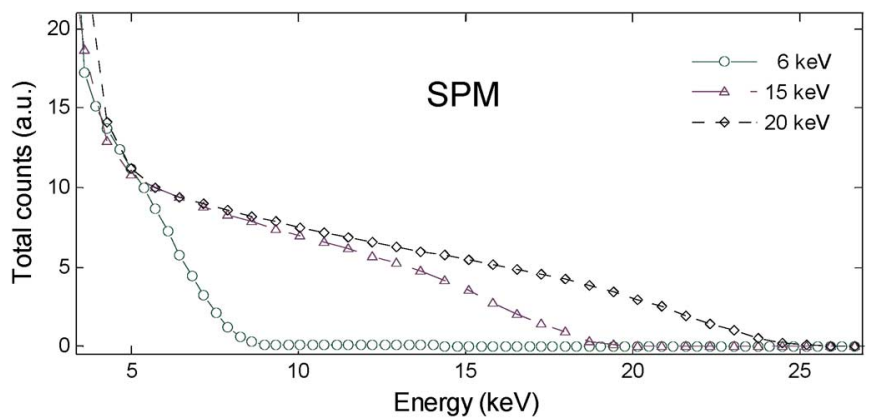

(a)

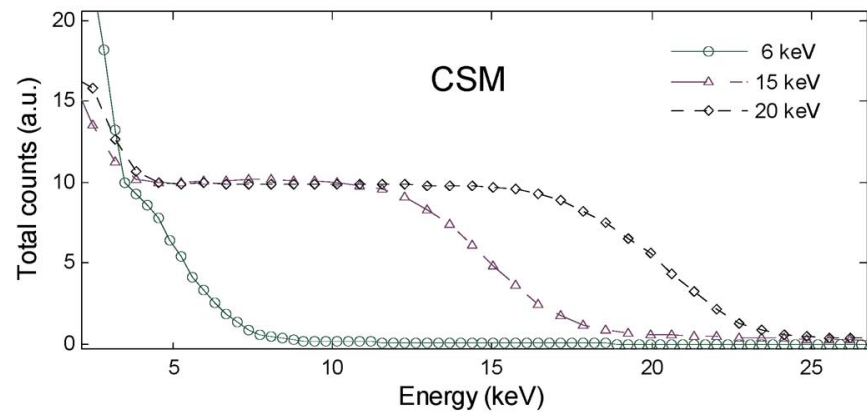

(b)

Fig. 7. Global S-curves for $6 \mathrm{keV}, 15 \mathrm{keV}$ and $20 \mathrm{keV}$ monochromatic X-ray beams for a Medipix3 operated in Single Pixel Mode (a) and in Charge Summing Mode (b). Charge sharing events contribute to the slope in the S-curves for Single Pixel Mode. In Charge Summing Mode, the flat region implies that charge-shared events are eliminated.

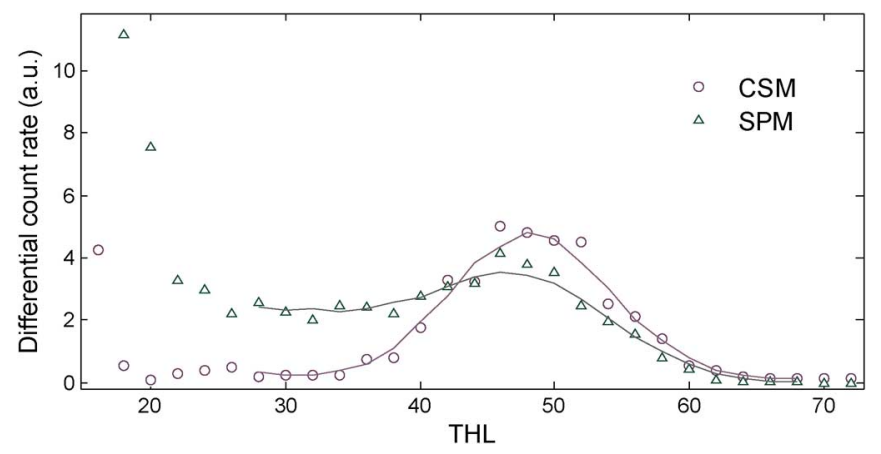

Fig. 8. Differential spectrum for Single Pixel Mode and Charge Summing Mode at $15 \mathrm{keV}$ X-rays. Single Pixel Mode spectrum shows collection of counts at low energies due to the charge shared effect between pixels. For Charge Summing Mode this effect is corrected.

design of the Medipix 3 chip eliminates event by event these low energy counts produced by charge-shared events.

For each differential spectrum, a Gaussian was fitted to the peak centroid, obtaining a correspondence between the THL value and the beam energy for each of the aforementioned eight energies. The linearity response of the detector for each mode under study is shown in Fig. 9. The detector is linear up to 30 $\mathrm{keV}$ for both SPM and CSM modes. A linear least-squares fit was applied to these points to find the calibration THL-energy for each mode. Results show that a step of 1 in THL corresponds to a step in energy of $0.360 \mathrm{keV}$ in SPM mode and $0.350 \mathrm{keV}$ in CSM mode, showing very good agreement between both operational modes. These conversion factors were then applied to the spectra. Table II shows the standard deviation value of the

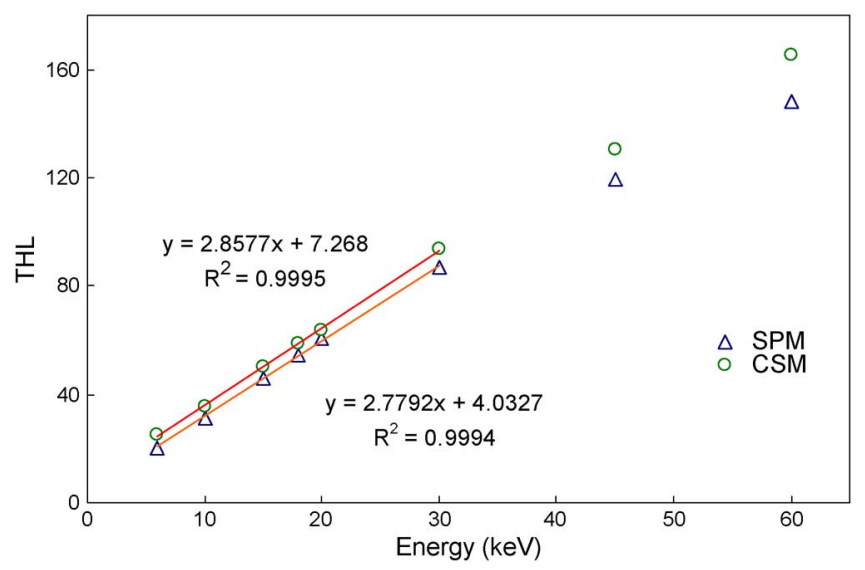

Fig. 9. Linearity response of the detector at different energies for Single Pixel Mode and Charge Summing Mode. Solid lines show the fits.

TABLE II

Standard DeViation VALUes $(\sigma)$ OF THE DifFerential SPECTRUM Fit AT DIFFERENT ENERGIES IN SPM AND CSM MODES

\begin{tabular}{c|cc|cc}
\hline \hline & \multicolumn{2}{|c|}{ SPM } & \multicolumn{2}{c}{ CSM } \\
\hline Energy (keV) & $\sigma(\mathrm{keV})$ & $\sigma(\mathrm{e}-\mathrm{rms})$ & $\sigma(\mathrm{keV})$ & $\sigma(\mathrm{e}-\mathrm{rms})$ \\
6 & 0.9 & 250 & 0.7 & 207 \\
15 & 1.6 & 454 & 1.4 & 380 \\
20 & 1.8 & 488 & 1.5 & 428 \\
\hline \hline
\end{tabular}

Gaussian fit performed on the differential spectrum for each of the energies.

\section{DEAD TIME}

The dead time of the electronic chain of the individual pixels (preamplifier, shaper, discriminator, counter) is an important parameter of the detector to evaluate. The measured counting rates have to be corrected in order to get the actual photon flux impinging on the pixel when the intensity is very high. An estimation of the dead time of the detector was obtained by exposing the detector to the unfocused direct beam. The counting rate of selected pixels was measured as aluminium attenuation foils, each of the same thickness, were placed in the beam. The resulting counting rates, referred to as output counting rate (ocr), were fitted with the well known paralyzable model [17], [18]. The free parameters were: the input count rate (icr) with no attenuation, the transmission of a single foil $(\theta)$ and the dead time of the detector $(\tau)$. The formula fitted was (1):

$$
o c r=i c r \cdot \theta^{N} \cdot e^{-i c r \cdot \theta^{N} \cdot \tau}
$$

where $N$ is the number of foils.

Dead time was evaluated for the SPM operational mode. In order to have the best estimate of the dead time, the model was fitted to seven pixels whose counting rate was higher than 200,000 counts per second when the beam was not attenuated. The transmission of a single aluminium foil was a parameter common to the seven sets of data and gave a value of 0.958 ; this is consistent with the thickness of the foils being $25 \mu \mathrm{m}$. The results for the dead times of the seven pixels were between 1.1 $\mu \mathrm{s}$ and $1.4 \mu \mathrm{s}$. Fig. 10. shows the counting rates for the seven 


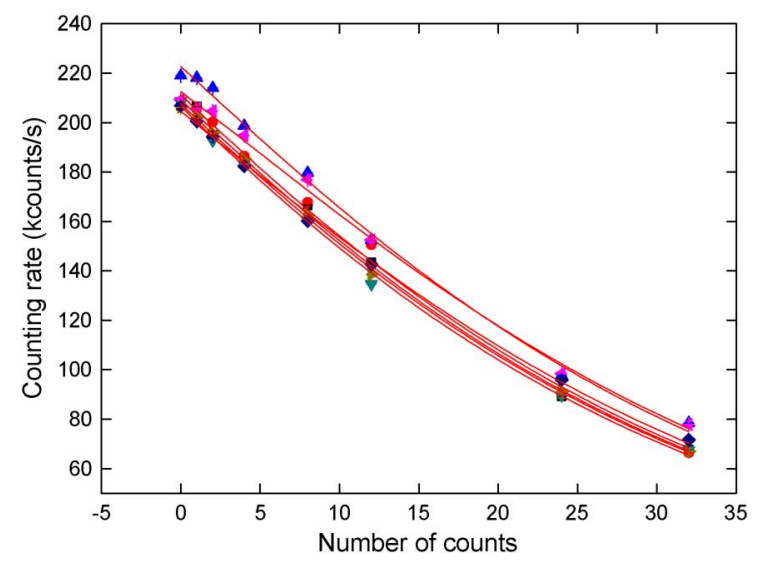

Fig. 10. Output counting rate vs number of foils for seven pixels with a counting rate greater than 200,000 counts/s when no foils were inserted. Each symbol corresponds to a pixel. Solid curves are the best fit of the dead time model for each pixel which enables to calculate the dead time parameter.

pixels as a function of the number of foils together with the fitted curves from the paralyzable model.

Formula (1) holds rigorously only for a random source. As explained at the beginning of Section III, the X-ray beam produced by DLS is structured. Thus it was checked up to what extent the classical paralyzable model was accurate in this case. The parameters resulting from the fit were fed into a model that takes into account the beam structure (see Appendix for details of the model). The maximum difference between the curve calculated with this model and the curve obtained with the classical paralyzable model was about $1.5 \%$. Therefore, there was no need to use a more detailed model.

\section{UNIFORMITY}

\section{A. Experimental Set-Up}

The uniformity of the response of the Medipix 3 detector was evaluated by flooding the detector with a uniform X-ray illumination. This uniform illumination was produced by the fluorescence emission from a $1 \mathrm{~mm}$ thick $\mathrm{Zr}$ sheet. An unfocused monochromatic X-ray beam of energy $18 \mathrm{keV}$ was used to produce fluorescence $16 \mathrm{keV} \mathrm{X}$-rays of energy. The detector was placed at a distance of $20 \mathrm{~cm}$ from the $\mathrm{Zr}$ target perpendicular to the incoming beam and flat-field images were acquired with an exposure time of $1 \mathrm{~s}$ resulting in a mean count rate of approximately 1000 counts per pixel per image.

\section{B. Flat-Field Correction Factors}

A flat-field image $\left(F F_{i j}\right)$ was obtained by summing 50 individual images. Flat-field correction factors $\left(K_{i j}\right)$ were computed from the flat-field image according to (2):

$$
K_{i j}=\frac{K}{F F_{i j}}
$$

where the scaling factor $K$ is the average pixel count calculated over the flat-field image $F F_{i j}$. This procedure was repeated for the SPM and CSM read-out modes and for two different threshold equalization masks (noise and $8 \mathrm{keV} \mathrm{X}$-rays). Pixels

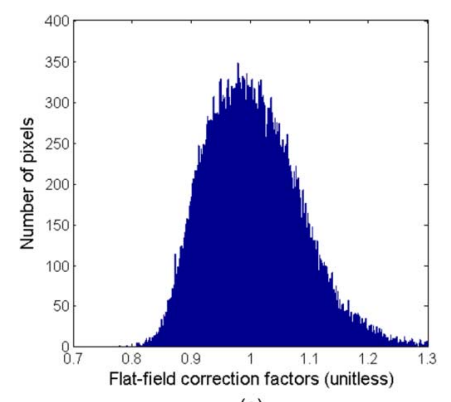

(a)

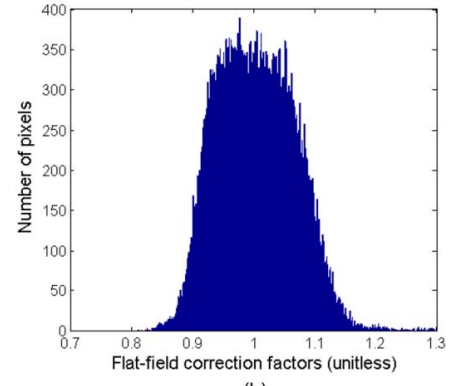

(b)
Fig. 11. Flat-field correction factors distribution in Single Pixel Mode for two different THL equalization masks: on noise (a) and on $8 \mathrm{keV} \mathrm{X-rays} \mathrm{(b).}$

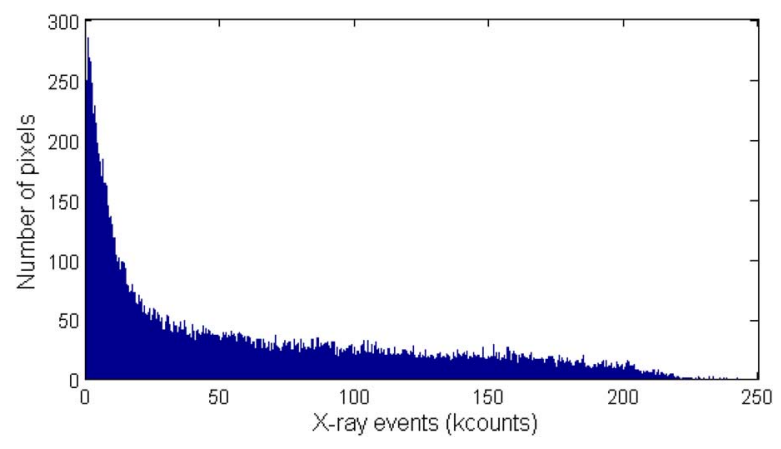

Fig. 12. Histogram of pixel counts in the sum of 50 flat-field images.

were considered as noisy and masked if their intensity was 50\% higher or lower than the mean flood-field image intensity.

In SPM mode, the number of masked pixels was 987 (1.51\% of active area) and 1000 (1.53\% of active area) for THL equalization on noise and on $8 \mathrm{keV} \mathrm{X-rays,} \mathrm{respectively.} \mathrm{The} \mathrm{distri-}$ bution of flat-field correction factors in SPM mode is shown in Fig. 11. for the two different THL equalizations. As expected these distributions are centred on 1 . The relative standard deviation of these two distributions was calculated to evaluate the dispersion in flat-field correction factors across the sensor. Relative standard deviations of $8.94 \%$ and $6.72 \%$ were measured for THL equalized on noise and on X-rays, respectively. This indicates an improvement in response uniformity when equalizing THL on X-rays.

In CSM mode, a total of 50 flat-field images with a mean count rate of $1000 \mathrm{X}$-rays/pixel/image were acquired and summed to produce the image shown in Fig. 12. The flat-field image obtained in CSM mode shows a strong noisy pattern due to the wrong allocation of X-ray counts by the detector. This is a consequence of the unexpected high pixel-to-pixel threshold variation already mentioned. Pixels with lower thresholds are assigned the hits more often than their neighbouring channels. Whereas some level of correction is possible, the image distortion introduced by the incorrect allocation of X-ray counts in the pixels cannot be corrected by a simple flat-field correction. A remapping of the pixel response is necessary based on more complex calibration procedures.

\section{Effect of Flat-Field Correction on Image SNR}

In SPM mode, the effectiveness of the flat-field correction was evaluated by applying flat-field correction factors $\left(K_{i j}\right)$ from 


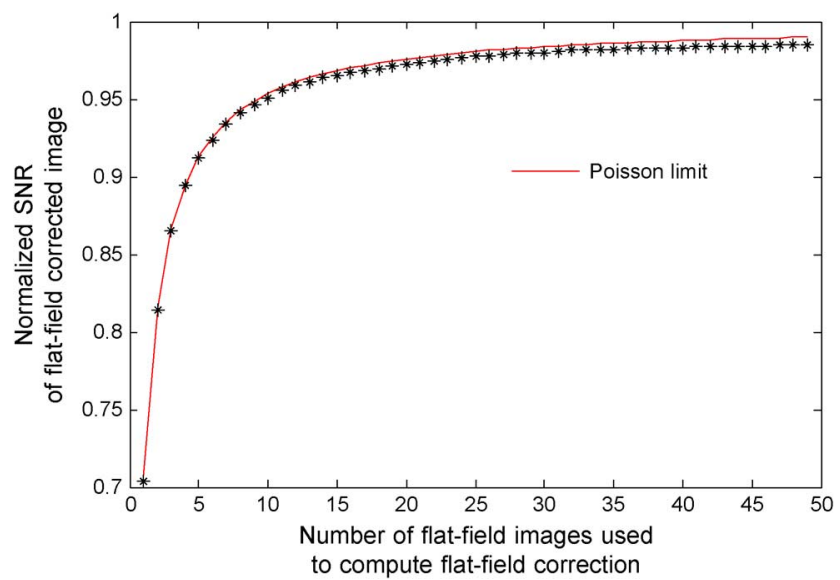

Fig. 13. Normalized SNR of a flat-field corrected image acquired in Single Pixel Mode as a function of the number of images used to compute the flat-field correction factors.

(2) to another flat-field image. The number of images used to compute flat-field correction factors was increased from 1 to 49. The signal-to-noise ratio (SNR) of the flat-field corrected image was normalized to the square root of the mean number of counts in the corrected image. The normalized SNR of the corrected image was compared to the ideal SNR which would be expected from Poisson statistics for a detector where pixel-to-pixel response fluctuations are determined only by X-ray photon noise.

Under this Poisson limit, the SNR of the flat-field image scales by $\sqrt{ } n$ and the variance of the corrected image, ratio of two independent random variables, is given by:

$$
\sigma^{2}=\sigma_{0}^{2}(1+1 / n)
$$

where $n$ is the number of images summed in the flat-field image used to compute flat-field correction factors and $\sigma_{0}$ is the variance of a single image, resulting from Poisson statistics only. Fig. 13. shows the SNR of a flat-field corrected image acquired in SPM mode as a function of the number of images used to compute the flat-field correction factors. Results show that the normalized SNR is closed to the ideal one, indicating that pixel-to-pixel variations observed in the histograms of flat-field correction factors are effectively removed by the flat-field correction process.

\section{Charge Collection}

\section{A. Charge Sharing Effect in Single Pixel Mode}

The micro-focused beam was used to scan two pixels along the shared side in steps of $5 \mu \mathrm{m}$ in order to study the effect of charge sharing. Threshold scans were performed at each position. When the beam is at the centre of the pixel, it collects all the charge as shown in Fig. 14(c). However, when the beam is on the edge of, or between, the pixels the charge is shared among them and the DAC value recorded (i.e. energy of the collected photons) is smaller than it should be, see Fig. 14(a) and (b).

\section{B. Micro-Focused Pixel Scan}

A $15 \mathrm{keV}$ micro-focused monochromatic X-ray beam was used to scan a pixel and its adjacent neighbours in order to study the

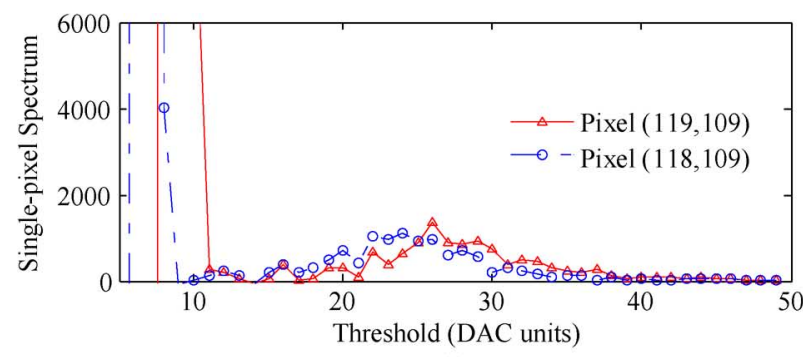

(a)

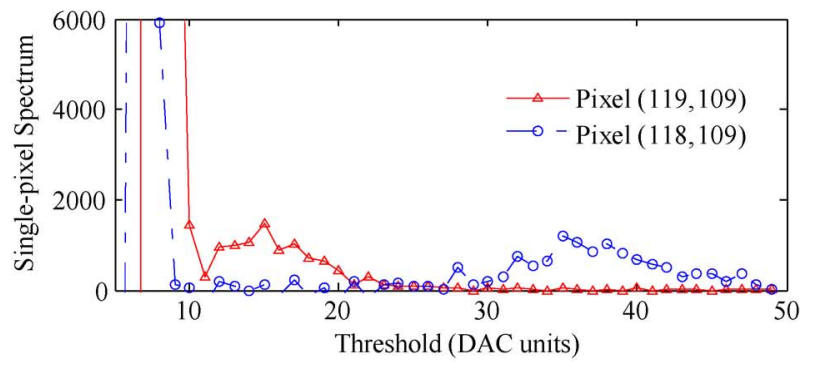

(b)

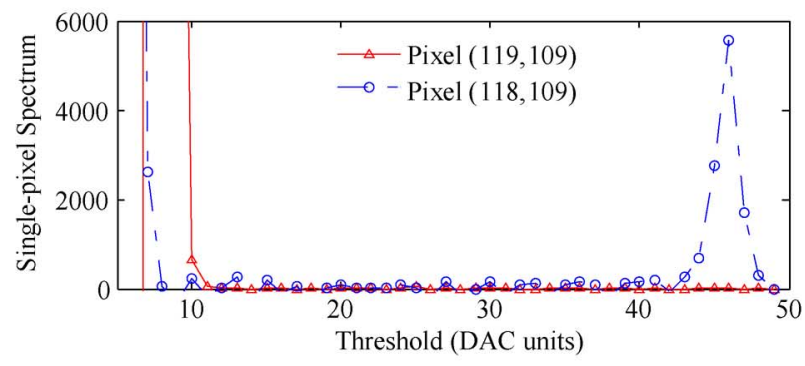

(c)

Fig. 14. Micro-focused scan perpendicular to the contact side of two pixels When the beam is at the edge, the charge is collected by both pixels independently, showing the charge sharing effect (a). As the scan moves to the center of one of the pixels the effect of charge sharing decreases (b). When the beam is at the center of the pixel (c), this pixel collects all the charge deposited by the photon and there is not charge sharing effect.

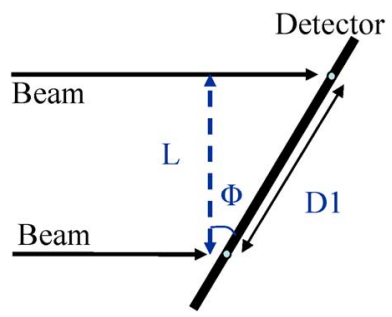

Fig. 15. Schematic of the alignment procedure.

charge acquisition process in SPM and CSM modes. In order to perform the experiments with the micro-focused beam, it was important to have aligned the detector perpendicular to the beam. The alignment procedure consisted of tilting the detector by an angle $\alpha$ and locating the pixel in the detector where the beam impinged. Then, the detector was moved a known distance along $x$ and the new beam impinging position was recorded. The distance between these two positions is defined as D1 (see Fig. 15.).

After tilting the detector $-\alpha$ degrees, distance D2 was obtained following the same procedure. If both distances are equivalent, the detector is aligned. In the case that they are different, (4) provides the correction angle $(\theta)$ to be applied [19].

$$
\tan \theta \tan \alpha=\frac{D 1-D 2}{D 1+D 2},
$$




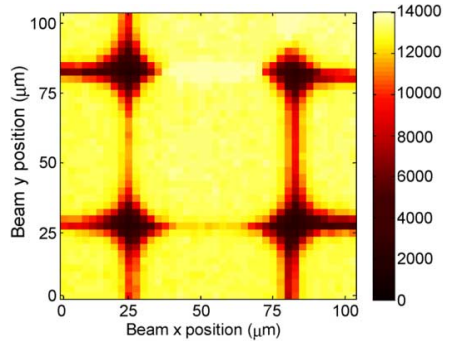

(a)

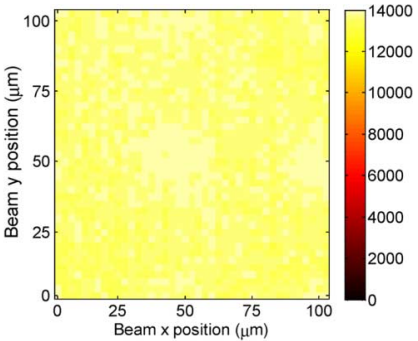

(b)
Fig. 16. A $100 \mu \mathrm{m}$ square map showing the sum of events recorded in all adjacent pixels as a function of beam position in $\mathrm{x}$ and $\mathrm{y}$ direction for the Medipix 3 operated on Single Pixel Mode (a) and in Charge Summing Mode (b) with a threshold set at $50 \%$ of the X-ray energy. In Single Pixel Mode a loss of counts at the pixel corners whereas in Charge Summing Mode there is a uniform response over the scanned area of the detector within $\pm 4 \%$.
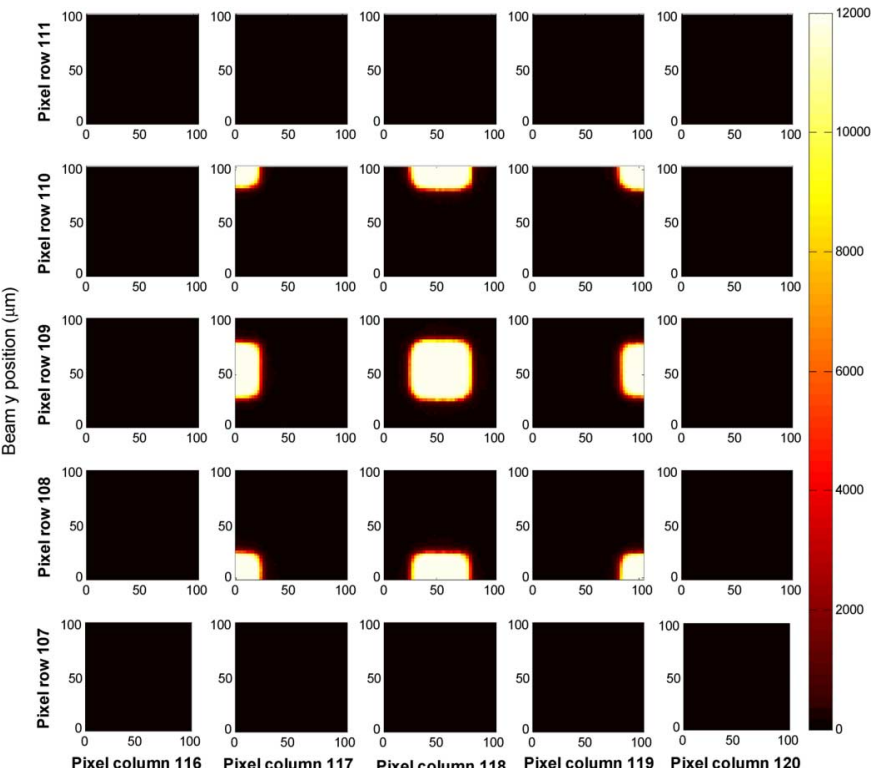

Pixel column 116 Pixel column 117 Pixel column 118 Beam $x$ position $(u \mathrm{~m})$

Fig. 17. Response of 25 individual pixels operated in Single Pixel Mode as a function of the beam position when a $100 \mu \mathrm{m}$ squared region (corresponding to 9 adjacent pixels centered on pixel $(118,109)$ ) was scanned. The image shows a correlation between the pixel that was scanned and the pixel that recorded the event.

The same procedure was followed to align the detector with respect to the other rotation plane $(\beta)$. The scan was performed with the pencil beam over an area of $100 \mu \mathrm{m} \times 100 \mu \mathrm{m}$ (centered on a pixel of the detector) in $2.5 \mu \mathrm{m}$ steps in both $x$ and $y$ directions by using the motorized detector table. The THL threshold was set at half the energy of the beam. An external TTL pulse from the beamline instrumentation triggered the detector acquisition.

The study of the charge sharing effect showed that it disappears for all energies when working in CSM mode (see Fig. 7). Moreover, when the chip was working in SPM mode, there was a loss of counts at the pixel corners (see Fig. 16(a)), whereas in CSM mode, there was a uniform collection of the charge for the whole detector area as it is shown in Fig. 16(b). However, when analyzing the process of recording photons for each readout mode, it was observed that whereas in SPM mode each
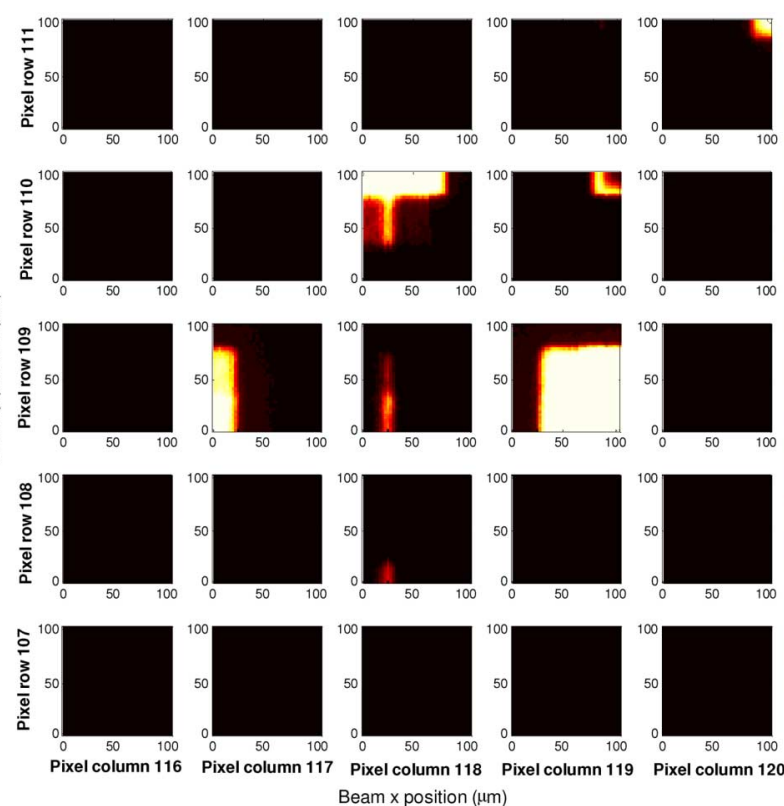

Fig. 18. Response of 25 individual pixels operated in Charge Summing Mode as a function of the beam position when a $100 \mu \mathrm{m}$ squared region (corresponding to 9 adjacent pixels centered on pixel $(118,109)$ ) was scanned. The image shows a mismatch between the pixels that were irradiated and the pixels that collected the charge. Even pixels that were not irradiated recorded events, c.f. pixel $(120,111)$.
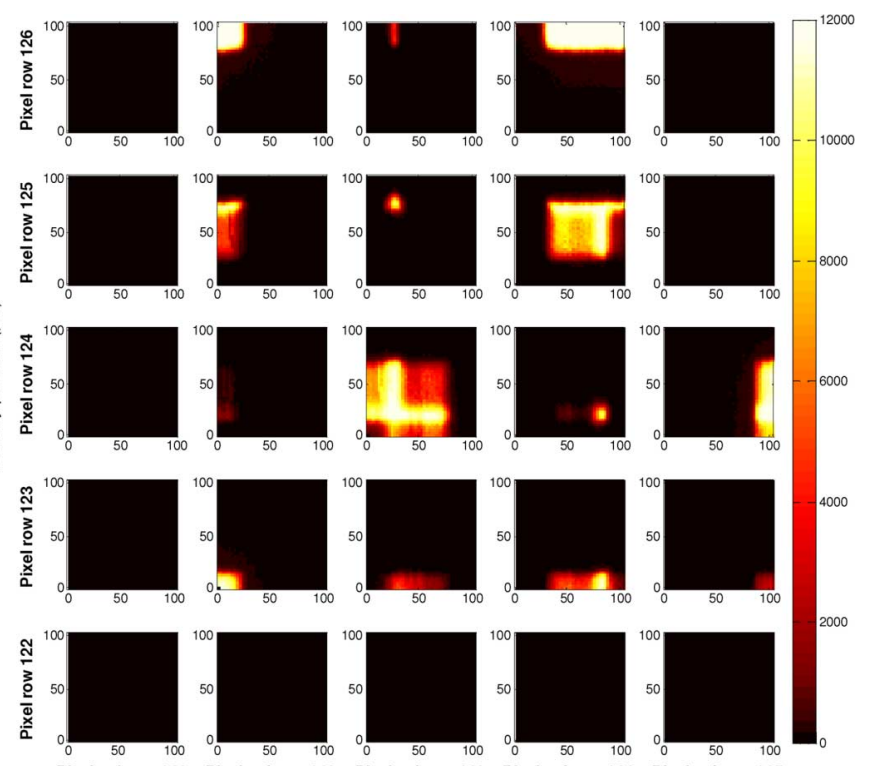

Pixel column 141 Pixel column 142 Pixel column 143 Pixel column 144 Pixel column 145 Beam $\times$ position $(\mu \mathrm{m})$

Fig. 19. Response of 25 individual pixels operated in Charge Summing Mode as a function of the beam position when a $100 \mu \mathrm{m}$ squared region (corresponding to 9 adjacent pixels centered on pixel $(143,123)$ ) was scanned. The mismatch between the irradiated pixel and the charge-collecting pixel was observed even for pixels that were not being irradiated (c.f. pixels $(144,126)$ or $(145,124))$. The charge-collection pattern varied with the region scanned, see Fig. 18.

pixel recorded the photons that impinged on it (see Fig. 17.), in CSM mode photon hits were wrongly allocated to their neighbours pixels as shown in Fig. 18 and Fig. 19. The mismatch between the irradiated pixel and the collecting pixel was observed even for pixels that were not being irradiated (c.f. pixels 
$(144,126)$ or $(145,124)$ in Fig. 19.). The structure of the collection readout in CSM varied with the region scanned, as it was observed when comparing the CSM mode readout for two different scan regions: one centered on pixel $(118,109)$ which is shown in Fig. 18.; and the other centered on pixel $(143,124)$ which is shown in Fig. 19. This effect was due to the unexpectedly aforementioned high threshold variation which leads the arbitration unit to assign the hit to the pixel with the lowest threshold in the region.

\section{CONCLUSION}

These are the first measurements taken of a Medipix3 chip bump-bonded to a silicon sensor at a synchrotron light source. The Medipix 3 chip is the first large mixed-mode pixel detector chip to be designed and produced in $0.13 \mu \mathrm{m}$ CMOS. New functionalities in the front-end architecture of Medipix 3 are implemented. Among them, the new CSM operating mode aimed at eliminating charge-shared events between adjacent pixels. In this work, the new CSM readout mode was studied and compared to the conventional SPM readout mode. Experiments show that in SPM mode, the pixel-to-pixel threshold dispersion improves when the threshold equalization is done on X-rays instead on noise. This leads to an improvement in the uniformity response of the detector, since the threshold equalization with $\mathrm{X}$-rays compensates for gain variation between pixels whereas equalization on noise compensates only for offset variation between pixels. Results of the energy spectra in Section V demonstrate that the CSM mode corrects the charge sharing effect seen in the SPM mode. Therefore, in CSM mode there is a uniform detection of the incident charge across the detector whereas in SPM mode the detector shows a loss in detection efficiency near pixel edges due to the charge sharing effect (Fig. 16). However in CSM mode, and independently of the threshold equalization performed, there is a large dispersion in the pixel response of the chip resulting in an incorrect allocation of X-rays counts to the pixels. Because of this, the flat-field image obtained shows a strong noise pattern. A remapping of the pixel response is necessary for this operational mode based on more complex calibration procedures. Finally, in both SPM and CSM modes, an unexpectedly high pixel-to-pixel threshold variation has been observed and this explains the erroneous hit allocation observed in CSM mode. This pixel-to-pixel mismatch is being investigated by the design team and will be corrected in a future version of the chip.

\section{APPENDIX}

After a detector counts one event, the amount of time that the detector is not able to count other events is defined as dead time. In a paralyzable detector any events occurring during the dead time are not recorded but they have an effect in the system since they prolong the amount of time that the detector is not receptive. This is the case considered for our system. Formula (1) provides the dead time correction for a paralyzable detector with a random source. However, synchrotrons are not completely a random source (see Section III) as it has been described by other authors [20], [21]. This approach and the use of MATHEMATICA code enabled the dead time to be calculated.
As the photons arrive in flashes $10 \mathrm{ps}$ long, the arrival of one or more photons cannot be distinguished. If at least one photon arrives, it will give rise to a count irrespective of how many others arrive. Therefore for each bunch of electrons, the probability that at least an event occurs is given by $p_{e}$ and by $\bar{p}_{e}=1-p_{e}$, the probability when no event occurs. An event occurring during the transit of a particular bunch of electrons can be counted only if the detector is idle. If the detector status and the fact that an event occurs are independent, then the probability to have one count is the product of the two probabilities. The dead time correction can then be derived for each bunch of electrons by calculating the probability that the detector is idle. The detector is idle if no events occurred during the $n$ bunches that transited within a period equal to the dead time. Therefore if in a period equal to the dead time, $n_{j}$ bunches transit before the bunch $j$, the probability that the detector is idle is given by expression (5):

$$
P_{j}(i d l e)=\left(\bar{p}_{e}\right)^{n_{j}},
$$

using the fact that events for each bunch are independent in the derivation of the last expression. The Poisson statistics for $p_{e}$ provide the final information necessary to derive the dead time correction. The difference between the curve calculated with this model and the curve obtained with the classical paralyzable model described in Section $\mathrm{V}$ was about $1.5 \%$.

\section{ACKNOWLEDGMENT}

This work was carried out in the framework of the Medipix3 Collaboration and the authors would like to acknowledge the other Collaboration members for valuable assistance, comments and support. In particular, colleagues from the Institute of Experimental and Applied Physics in Prague are thanked for providing the read-out electronics and the updated Pixelman software. Furthermore, the authors would like to acknowledge Mr. Brian Willis for his invaluable help in preparing the housing for the assemblies and their mounting on the beamline.

\section{REFERENCES}

[1] R. Lewis, "Position sensitive detectors for synchrotron radiation studies: The tortoise and the hare?,' Nucl. Instrum. Methods Phys. Res. A, vol. A513, pp. 172-177, 2003.

[2] P. Kraft, A. Bergamaschi, C. Brönnimann, R. Dinapoli, E. F. Eikenberry, H. Graafsma, B. Henrich, I. Johnson, M. Kobas, A. Mozzanica, C. M. Schlepütz, and B. Schmitt, "Characterization and calibration of PILATUS detectors,” IEEE Trans. Nucl. Sci., vol. 56, pp. 758-764, 2009.

[3] P. Pangaud, S. Basolo, N. Boudet, J.-F. Berar, B. Chantepie, J.-C. Clemens, P. Delpierre, B. Dinkespiler, K. Medjoubi, S. Hustache, M. Menouni, and C. Morel, "XPAD-S: A fast hyprid pixel readout chip for X-ray synchrotron facilities," Nucl. Instrum. Methods Phys. Res. A, vol. A591, pp. 159-162, 2008.

[4] J.-F. Berar, N. Boudet, P. Breugnon, B. Caillot, B. Chantepie, J.-C. Clemens, P. Delpierre, B. Dinkespiller, S. Godiot, C. Meessen, M. Menouni, C. Morel, P. Pangaud, E. Vigeolas, S. Hustache, and K. Medjoubi, "XPAD3 hybrid pixel detectors applications," Nucl. Instrum. Methods Phys. Res. A, vol. A607, pp. 233-235, 2009.

[5] Diamond Light Source synchrotron [Online]. Available: http://www. diamond.ac.uk

[6] J. Marchal, N. Tartoni, and C. Nave, "Synchrotron applications of pixel and strip detectors at Diamond Light Source," Nucl. Instrum. Methods Phys. Res. A, vol. A604, pp. 123-126, 2009. 
[7] R. Ballabriga, M. Campbell, E. Heijne, X. Llopart, L. Tlustos, and W. Wong, "MEDIPIX3: A $64 \mathrm{k}$ pixel detector readout chip working in single photon counting mode with improved spectrometric performance," Nucl. Instrum. Methods Phys. Res. A, 2010, 10.1016/j.nima. 2010.06.108, accepted for publication.

[8] R. Ballabriga, M. Campbell, E. H. M. Heijne, X. Llopart, and L. Tlustos, "The Medipix3 prototype, a pixel readout chip working in single photon counting mode with improved spectrometric performance," IEEE Trans. Nucl. Sci., vol. 54, pp. 1824-1829, 2007.

[9] B16 Beamline Plan Diamond Light Source, 2010 [Online]. Available: http://www.diamond.ac.uk/Home/Beamlines/B16.html

[10] Z. Vykydal, J. Jakubek, and S. Pospisil, "USB interface for Medipix2 pixel device enabling energy and position-sensitive detection of heavy charged particles," Nucl. Instrum. Methods Phys. Res. A, vol. 563, pp. 112-115, 2006.

[11] Institute of Experimental and Applied Physics, Czech Technical University in Prague [Online]. Available: http://www.utef.cvut.cz

[12] J. Marchal, "Theoretical analysis of the effect of charge-sharing on the detective quantum efficiency of single-photon counting segmented silicon detectors," J. Inst., vol. 5, p. P01004, 2010.

[13] D. Pennicard, J. Marchal, C. Fleta, G. Pellegrini, M. Lozano, C. Parkes, N. Tartoni, D. Barnett, I. Dolbnya, K. Sawhney, R. Bates, V. O'Shea, and V. Wright, "Synchrotron tests of a 3D Medipix2 x-ray detector," IEEE Trans. Nucl. Sci., vol. 57, pp. 387-394, 2010.

[14] E. N. Gimenez, D. Maneuski, A. Mac Raighne, C. Parkes, R. Bates, V. O'Shea, C. Fleta, G. Pellegrini, M. Lozano, L. Alianelli, K. J. S. Sawhney, J. Marchal, and N. Tartoni, "3D Medipix2 detector characterization with a micro-focused X-ray beam," Nucl. Instrum. Methods Phys. Res. A, 2010, 10.1016/j.nima.2010.06.140, accepted for publication.
[15] R. Ballabriga, The Design and Implenatation in 0.13 um CMOS of an Algorithm Permitting Spectroscopic Imaging With High Spatial Resolution for Hybrid Pixel Detectors, CERN-THESIS-2010-055.

[16] R. Ballabriga, W. Wong, and X. Llopart, Medipix3 Manual v1.9 2009 [Online]. Available: http://medipix.web.cern.ch/MEDIPIX

[17] G. F. Knoll, Radiation Detection and Measurement. New York: Wiley, 2000.

[18] R. D. Evans, The Atomic Nucleus. Melbourne, FL: Krieger, 1982.

[19] A. Mac Raighne, K. C. Akiba, L. Alianelli, M. Artuso, R. Bates, F. Bayer, J. Buytaert, P. Collins, M. Crossley, L. Eklund, C. Fleta, A. Gallas, M. Gandelman, M. Gersabeck, E. N. Gimenez, V. Gligorov, T. Huse, M. John, L. F. Llin, M. Lozano, D. Maneuski, J. Marchal, T. Michel, M. Nicol, G. Pellegrini, D. E. Perira, R. Plackett, V. O'Shea, C. Parkes, E. Rodrigues, K. J. S. Sawhney, N. Tartoni, and P. Vazquez, "Synchrotron tests of 3D Medipix2 and TimePix x-ray detectors," in Proc. NSS-MIC Conf., 2009, 10.1109/NSSMIC.2009.5402093.

[20] J. E. Bateman, "The effect of beam time structure on counting detectors in SRS experiments," J. Synchrotron Radiat., vol. 7, pp. 307-312, 2000.

[21] D. A. Walko, D. A. Arms, and E. C. Landahl, "Empirical dead-time corrections for synchrotron sources," J. Synchrotron Radiat., vol. 15, pp. 612-617, 2008. 